\title{
Thermionic field emission at electrodeposited Ni-Si Schottky barriers
}

\author{
M.E. Kiziroglou ${ }^{\mathrm{a}, *}$, X. Li ${ }^{\mathrm{a}}$, A.A. Zhukov ${ }^{\mathrm{b}}$, P.A.J. de Groot ${ }^{\mathrm{b}}$, C.H. de Groot ${ }^{\mathrm{a}}$ \\ ${ }^{a}$ School of Electronics and Computer Science, University of Southampton, SO17 1BJ Southampton, UK \\ ${ }^{\mathrm{b}}$ School of Physics and Astronomy, University of Southampton, SO17 1BJ Southampton, UK
}

\section{A R T I C L E I N F O}

\section{Article history:}

Received 5 December 2007

Received in revised form 4 February 2008

Accepted 1 March 2008

Available online 23 April 2008

The review of this paper was arranged by Prof. A. Zaslavsky

\section{Keywords:}

Schottky barriers

Tunneling

Spintronics

\begin{abstract}
A B S T R A C T
Current transport at Schottky barriers is of particular interest for spin injection and detection in semiconductors. Here, electrodeposited $\mathrm{Ni}$-Si contacts are fabricated and the transport mechanisms through the formed Schottky barrier are studied. Highly doped $S i$ is used to enable tunneling currents. $I-V, C-V$ and low-temperature $I-V$ measurements are performed and the results are interpreted using tunneling theory for Schottky barriers and recent models for spatially distributed barrier heights. It is shown that, contrary to the case of lowly doped Si where thermionic emission dominates, tunneling is the dominant mechanism for reverse and low forward bias for highly doped $\mathrm{Si}$. An exponential reverse bias $I-V$ behavior with negative temperature coefficient is reported. An explanation can be found on the rapid decrease of the reverse bias $I-V$ slope with temperature predicted by Padovani and Stratton for thermionic field emission in conjunction with the increase of the Schottky barrier height with temperature suggested for spatially distributed barrier heights.
\end{abstract}

(c) 2008 Elsevier Ltd. All rights reserved.

\section{Introduction}

Spin injection from a magnetic metal into a semiconductor requires spin conservation during the injection of the electrons. It is particularly interesting because it allows the integration of magnetic devices with microelectronics. The conductivity mismatch problem has been shown to prevent ohmic contacts from being used for spin injection [1,2]. Instead, Schottky barriers (SBs) and Ferromagnet (FM)/Insulator/Semiconductor contacts have been used $[3,4]$. A FM/Si/FM spintronic device has been proposed by Bratkovsky and Osipov using the SBs for injection and extraction of spin polarized electrons [5]. A detailed understanding of the transmission mechanisms in the SBs is required for the fabrication of such devices.

Thermionic emission (TE) over a SB is believed to be unsuitable for spin injection and detection because reverse bias electron transmission occurs at energies well above the Fermi level [6]. Image force lowering causes the top of the barrier to occur inside the semiconductor rather than at the interface. Thus, electrons emitted over the barrier are already well in the semiconductor where the density of states is not spin-resolved. On the other hand, thermionic field emission takes place at a lower energy. The electrons still tunnel within the semiconductor [7] but from a vicinity much closer to the metal, where spin-resolved density of states still ap-

\footnotetext{
* Corresponding author. Present address: Imperial College, London. Tel.: +44 7961034114; fax: +44 2075946308.

E-mail address: m.kiziroglou@ic.ac.uk (M.E. Kiziroglou).
}

plies and can provide spin-dependent transmission. Therefore, thermionic field emission through Schottky barriers is promising for spin injection/detection. This has already been demonstrated in metal/GaAs structures in optical detection experiments [8].

Electrodeposited Ni-Si SBs with lowly doped $\mathrm{Si}(\mathrm{nSi})$ exhibit a high quality Schottky barrier with very low leakage [9]. Analysis using TE models has revealed a large SB height. In the work presented here electrodeposited $\mathrm{Ni}-\mathrm{Si}$ contacts using highly doped $\mathrm{Si}(\mathrm{n}+\mathrm{Si})$ to activate tunneling were fabricated and characterized. The results are analyzed and compared with those of lowly doped $\mathrm{Si}$, with the thermionic emission theory and with the predictions of Padovani and Stratton for tunneling through SBs [10]. The potential of electrodeposited Schottky contacts for spin injection and detection is discussed taking into account forward and reverse bias Thermionic field emission (TFE).

\section{Thermionic and thermionic field emission}

According to the thermionic emission theory for SBs the current density $\left(J_{\mathrm{TE}}\right)$ versus applied potential $(V)$ characteristic is described by the following equations [11]:

$J_{\mathrm{TE}}=J_{\mathrm{S}, \mathrm{TE}} \cdot\left(\mathrm{e}^{\frac{V}{V_{\mathrm{t}}}}-1\right)$

$J_{\mathrm{S}, \mathrm{TE}}=A^{*} \cdot T^{2} \cdot \mathrm{e}^{-\frac{\phi_{B n}}{V_{\mathrm{t}}}}$

where $J_{\mathrm{S}, \mathrm{TE}}$ is the thermionic emission saturation current density, $T$ is the absolute temperature, $V_{\mathrm{t}}=k T / q$ (here $q$ is the elementary charge and $k$ is the Boltzmann constant) and $A^{*}=120 \mathrm{~m}^{*} / \mathrm{m}$ 
$\left(A \mathrm{~cm}^{-2} \mathrm{~K}^{-2}\right.$ ) is the effective Richardson constant with $m^{*}$ and $m$ being the effective and the free electron mass respectively. $\phi_{B n}$ is the Schottky barrier height including image force lowering:

$\phi_{B n}=\phi_{\mathrm{B}, 0}-\sqrt{\frac{q \cdot E_{\mathrm{max}}}{4 \pi \varepsilon_{\mathrm{s}}}}$

where $\phi_{\mathrm{B}, 0}$ is the barrier height without image force lowering and $\varepsilon_{\mathrm{S}}$ is the dielectric constant of the semiconductor. $E_{\max }$ is the maximum electric field in the depletion region given by

$E_{\max }=\sqrt{\frac{2 q \cdot N_{\mathrm{D}}}{\varepsilon_{\mathrm{s}}} \cdot\left(\phi_{\mathrm{B}, 0}-V-\xi-V_{\mathrm{t}}\right)}$

Here, $N_{\mathrm{D}}$ is the semiconductor doping concentration and $\xi=V_{\mathrm{t}} \cdot \ln \left(N_{\mathrm{C}} / N_{\mathrm{D}}\right) . N_{\mathrm{C}}$ is the effective density of states at the bottom of the conduction band.

Recent models for thermionic emission assume a spatial distribution of the barrier height to take interface inhomogeneities into account [12]. If $\overline{\phi_{\mathrm{B}, 0}}$ and $\sigma_{\mathrm{s}}$ are the mean value and the standard deviation of the distribution respectively, the barrier height will have a temperature dependence which can be described by the following equation:

$\phi_{\mathrm{B}, 0}=\overline{\phi_{\mathrm{B}, 0}}-\frac{q \cdot \sigma_{\mathrm{s}}^{2}}{2 \cdot k \cdot T}$

Tunneling at Schottky barriers can occur either at the Fermi level (field emission) or at an energy above Fermi level (TFE). For the Si doping level and the temperature range used in this work, no significant field emission is expected [11]. Therefore, only TFE is considered in the analysis. The analytical expressions for forward $\left(J_{T F E, f}\right)$ and reverse $\left(J_{\mathrm{TFE}, \mathrm{r}}\right)$ bias TFE current, as adapted from Padovani and Stratton [10] are given below:

$J_{\text {TFE, } \mathrm{f}}=J_{\mathrm{s}, \mathrm{TFE}, \mathrm{f}} \cdot \mathrm{e}^{\frac{V}{E_{0}}}$

$J_{\mathrm{TFE}, \mathrm{r}}=J_{\mathrm{s}, \mathrm{TFE}, \mathrm{r}} \cdot \mathrm{e}^{\frac{V}{t}}$

where

$$
\begin{aligned}
& J_{\mathrm{s}, \text { TFE, } \mathrm{f}}=\frac{A^{*} \cdot T^{2} \cdot \sqrt{\pi E_{00}}}{V_{\mathrm{t}} \cdot \cosh \left(E_{00} / V_{\mathrm{t}}\right)} \cdot \mathrm{e}^{-\frac{\phi_{B n}}{E_{0}}} \cdot \sqrt{\phi_{B n}-V-\xi} \cdot\left(\frac{N_{\mathrm{c}}}{N_{\mathrm{d}}}\right)^{\frac{V_{\mathrm{t}}}{E_{0}}-1} \\
& J_{\mathrm{s}, \mathrm{TFE}, \mathrm{r}}=\frac{A^{*} \cdot T^{2} \cdot \sqrt{\pi E_{00}}}{V_{\mathrm{t}} \cdot \cosh \left(E_{00} / V_{\mathrm{t}}\right)} \cdot \mathrm{e}^{-\frac{\phi_{B n}}{E_{0}}} \cdot \sqrt{\phi_{B n}-V \cdot \cosh ^{2}\left(E_{00} / V_{\mathrm{t}}\right)} \\
& E_{0}=\frac{E_{00}}{\tanh \left(\frac{E_{00}}{V_{\mathrm{t}}}\right)} \\
& \varepsilon^{\prime}=\frac{E_{00}}{E_{00} / V_{\mathrm{t}}-\tanh \left(E_{00} / V_{\mathrm{t}}\right)} \\
& E_{00}=\frac{h}{2} \cdot \sqrt{\frac{N_{\mathrm{d}}}{m^{*} \epsilon_{\mathrm{s}}}}
\end{aligned}
$$

\section{Experimental procedure}

$\mathrm{Ni}-\mathrm{n}+\mathrm{Si}$ contacts were fabricated by electrodeposition on $\mathrm{n}$ type, $\langle 100\rangle$ Si wafers with resistivity $0.01-0.02 \Omega \mathrm{cm}$. For comparison, $\mathrm{Ni}-\mathrm{nSi}$ contacts were also fabricated. To avoid a large series resistance in the latter case, the $\mathrm{nSi}$ was grown epitaxially on highly doped $\mathrm{Si}$ wafers ( $\mathrm{n}$ on $\mathrm{n}+$ ). The $\mathrm{n}+\mathrm{Si}$ resistivity was again $0.01-0.02 \Omega \mathrm{m}$ while the $\mathrm{nSi}$ resistivity was $0.9-1.0 \Omega \mathrm{cm}$. A $20 \mathrm{~nm}$ thick layer of $\mathrm{SiO}_{2}$ was thermally grown on the front side. Circular and square patterns of sizes from $0.1 \mathrm{~mm}$ to $1.5 \mathrm{~mm}$ were transferred to the $\mathrm{SiO}_{2}$ layer by conventional lithography. Al Ohmic back contacts were created by evaporation and annealing for 30 min at $450{ }^{\circ} \mathrm{C}$ in $\mathrm{H}_{2} / \mathrm{N}_{2}$ to allow for electrical measurements. Subsequently, after a standard RCA cleaning step (Radio Corporation of America, $\mathrm{H}_{2} \mathrm{O}_{2} / \mathrm{NH}_{4} \mathrm{OH}$ followed by $\mathrm{H}_{2} \mathrm{O}_{2} / \mathrm{HCl}$ ) and a 20:1 buffered HF dip for $30 \mathrm{~s}$, electrodeposition of Ni directly on $\mathrm{Si}$ was performed.

For electrodeposition, a $\mathrm{Ni}$ sulphate bath and an Autolab AUT72032 potentiostat three electrode system with a Pt counter electrode and a saturated calomel reference electrode were used [13]. The deposition potential was $-1.1 \mathrm{~V}$ (against the reference electrode). A pulse of $-1.7 \mathrm{~V}$ was applied for $0.4 \mathrm{~s}$ just before the deposition stage to form a uniform Ni nucleation on Si which led to smoother deposition [14]. A metal overlap structure was formed

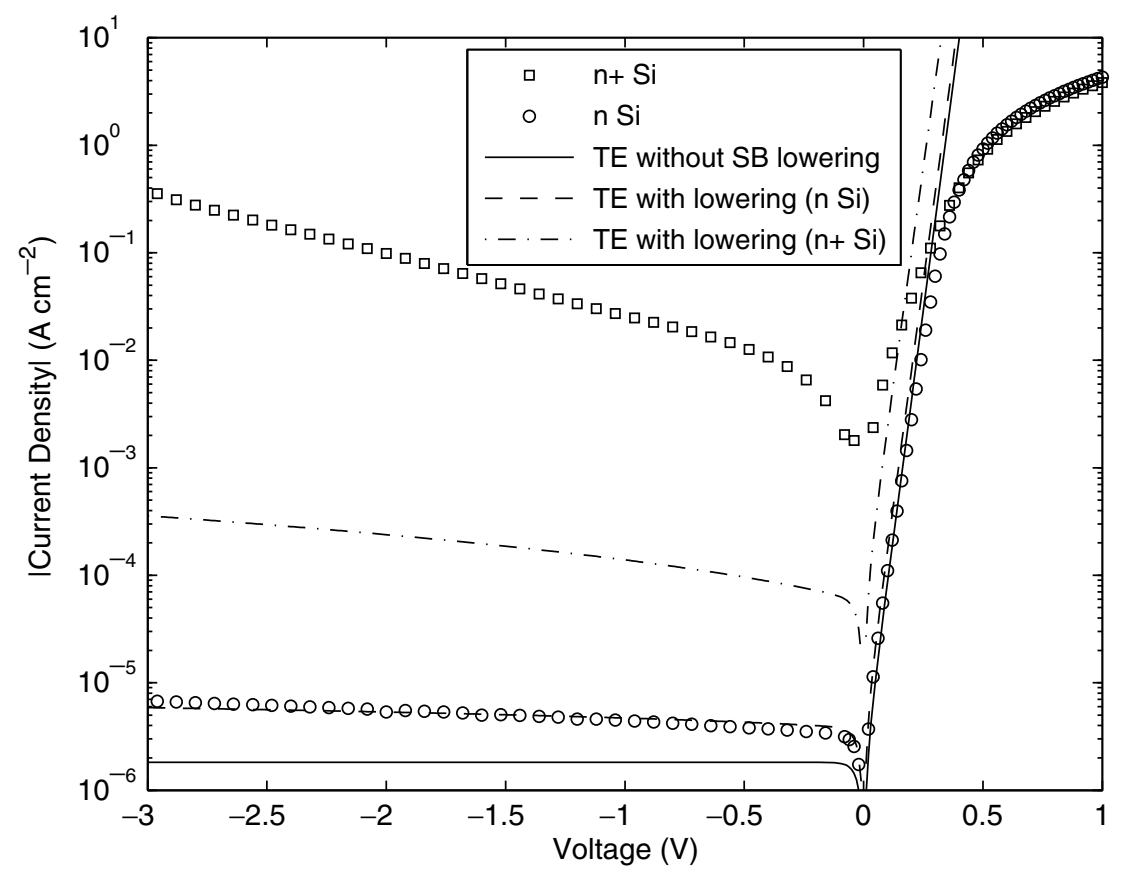

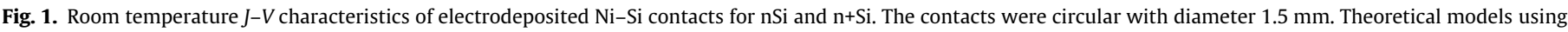
the SB height extracted from the forward bias of the Ni-nSi characteristic are also shown. 
at the edges suppressing the edge leakage currents. Hence, the formation of a guard ring was not required for these SBs [15]. SBs with Ni layer thicknesses from $200 \mathrm{~nm}$ to $400 \mathrm{~nm}$ were fabricated but no variation of the SB parameters with thickness was observed.

$I-V$ and $C-V$ characteristics measurements were performed using a Hewlett Packard 4155 A semiconductor parameter analyzer and a Hewlett Packard $4280 \mathrm{~A}, 1 \mathrm{MHz}, \mathrm{C}$ Meter/C-V plotter. Low temperature $I-V$ measurements down to $86 \mathrm{~K}$ were performed using a Bio-Rad DL 4960 cryostat temperature controller.

\section{Results and discussion}

Room temperature current density $(J)$ versus voltage $(V)$ measurements of a $\mathrm{Ni}-\mathrm{n}+\mathrm{Si}$ contact are shown as squares in Fig. 1. For comparison, $J-V$ measurements of a Ni-n on $n+S i$ contact are also shown (circles in Fig. 1). The use of $n+$ bulk Si for both cases assured the same series resistance as it is apparent from the concordance of the two experimental curves at forward bias higher than $0.4 \mathrm{~V}$.

For $\mathrm{nSi}$, a high quality rectifying behavior is observed. Thermionic emission can be assumed as the dominant transport mechanism, since the lowly doped n layer suppresses tunneling effects. By extrapolation from the exponential forward bias region, a SB height value of $\Phi_{\mathrm{b}, 0}=0.76 \mathrm{~V}$ is obtained. Here, image force lowering as described by Eq. (3) has been taken into account using the $N_{\mathrm{D}}$ value that corresponds to the resistivity of the n-Si. The fitting characteristic is shown as a dashed line in Fig. 1. The theoretical TE characteristic for the same $\Phi_{b, 0}$ without lowering is also shown as a solid line in Fig. 1. The difference is more clear in the reverse bias region, demonstrating the excess current that can be attributed to image force lowering for nSi.

For $\mathrm{n}+\mathrm{Si}$, excess current is evident in the reverse bias and in the low forward bias, for voltages up to $0.3 \mathrm{~V}$. This current increase with doping concentration can be attributed either to the increase of the Schottky barrier (SB) image force lowering effect or to tunneling currents through the barrier. Generation - recombination effects can be ruled out since they decrease with increasing doping concentration.
The image force lowering effect expected for the $\mathrm{Ni}-\mathrm{n}+\mathrm{Si}$ contacts is given by Eq. (3), using the $n+S i$ doping concentration $\left(2.1 \mathrm{E} 18 \mathrm{~cm}^{-3}\right)$ and the $\Phi_{\mathrm{b}, 0}$ extrapolated from the forward bias of the nSi curve $(0.76 \mathrm{~V})$. The resulting curve is shown as a dash-dotted curve in Fig. 1. Although a significant $J$ increase could be caused by image force lowering, it is not enough to explain the excess currents for $n+S i$. The above considerations suggest tunneling as the dominant transport mechanism at electrodeposited $\mathrm{Ni}-\mathrm{n}+\mathrm{Si}$ contacts.

To further investigate this suggestion, low temperature measurements were performed as shown in Fig. 2. These measurements were taken with a temperature step of $10 \mathrm{~K}$. For clarity, only five curves are plotted in Fig. 2. In the forward bias, a minor increase of $\mathrm{J}$ with $\mathrm{T}$ is observed. In the low reverse bias, J increases with $\mathrm{T}$ as well. However, for temperatures below $250 \mathrm{~K}$, at around $-1 \mathrm{~V}$ to $-1.5 \mathrm{~V}$ a sudden slope increase is observed indicating a switch of the dominant transport mechanism. For reverse bias larger than $-1.5 \mathrm{~V}$, the temperature coefficient becomes negative and a cross-over region is observed.

Eq. (7) shows that the reverse bias TFE current $J_{\mathrm{TFE}, \mathrm{r}}$ has an exponential dependence on the applied potential $V$, with a slope of $1 / \varepsilon^{\prime}$. Given that $\varepsilon^{\prime}$ increases with increasing temperature (Eq. (11)), the slope is higher for lower temperatures and hence, $J_{\mathrm{TFE}, \mathrm{r}}-V$ curves for different temperatures may eventually give a cross-over point. For voltages beyond this point the current will be higher for lower temperatures. Therefore, the cross-over and negative temperature coefficient observed in Fig. 2 are consistent with the predictions of Padovani and Stratton for reverse bias TFE current voltage characteristics [10].

This effect is not observed in the experimental results of Padovani and Stratton. An explanation can be obtained by taking into account the rapid decrease of the saturation current $J_{\mathrm{s}, \mathrm{TFE}, \mathrm{r}}$ with decreasing temperature suggested in Eq. (9). This effect increases the voltage at which the cross over occurs beyond the range of measurements. In contrast, electrodeposited Schottky barriers exhibit a spatial distribution of the SB height which results in a decreasing SB height with decreasing temperature [9]. Hence, the TFE saturation current $J_{\mathrm{s}, \mathrm{TFE}}$ is kept in levels high enough to bring the cross-over and negative temperature coefficient effects within

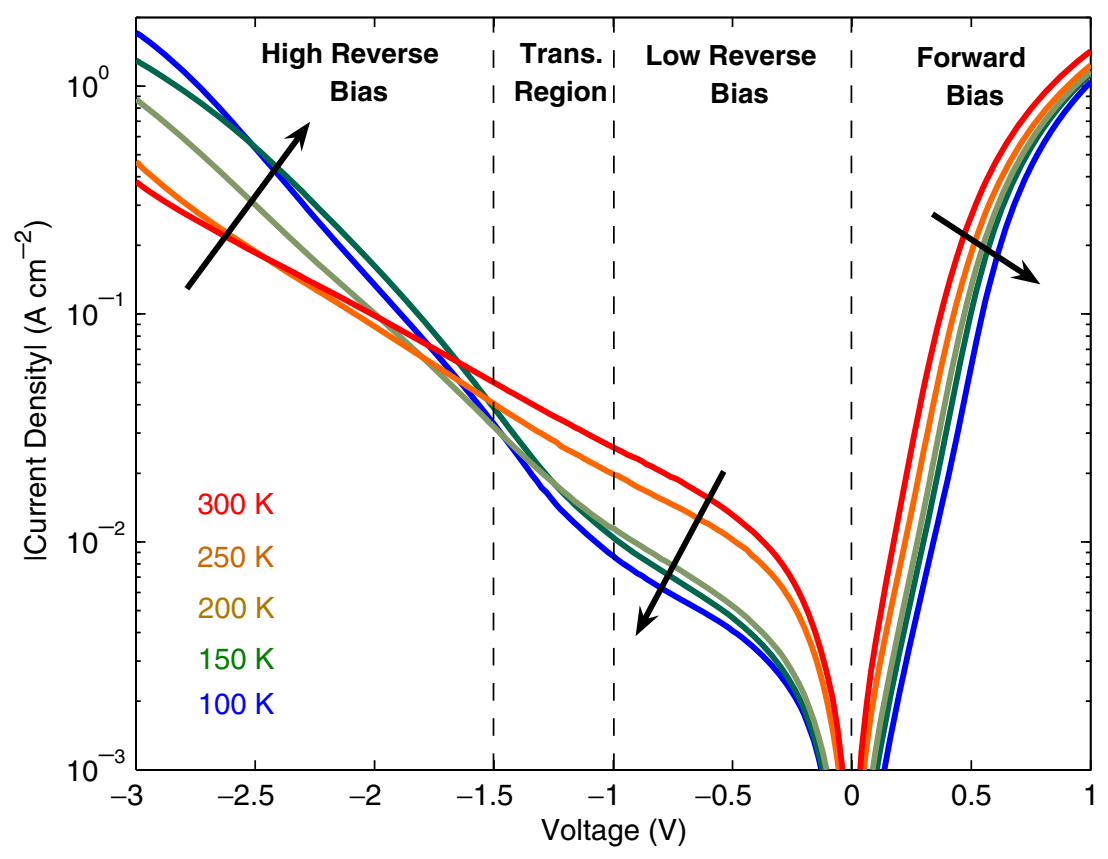

Fig. 2. Low temperature $J-V$ characteristics of an electrodeposited Ni-Si contact for $n+S i$. The contact was circular with diameter 1.5 mm. 
measuring range. To further support the above arguments, the experimental data are analyzed below, taking into account the TFE theory and the spatial distribution of the SB height.

\subsection{Forward bias}

By extension of the forward bias linear part of Fig. 2, from $0.1 \mathrm{~V}$ to $0.4 \mathrm{~V}$, to the $\mathrm{J}$ axis, the saturation current density is obtained for different temperatures. These measurements are plotted as filled circles in Fig. 3. For comparison, corresponding data for nSi are shown as open circles.

The increase of $J_{\mathrm{s}}$ with temperature for $\mathrm{n}+\mathrm{Si}$ is much smaller than that observed for the $\mathrm{nSi}$. This can be explained by comparing the theoretical temperature dependence of $J_{\mathrm{s}}$ for thermionic and thermionic field emission as given by Eqs. (2) and (8). While thermionic emission always gives a strong temperature dependence, thermionic field emission can be much less temperature dependent.

The experimental results for $\mathrm{nSi}$ suggest a spatial distribution of the SB height. A good fit is obtained by using the model of Werner and Guttler [12]. This fit is shown as a dashed line in Fig. 3. In analogy, a spatial SB height distribution can be assumed for the $n+S i$ contact and used in Eq. (8) for the thermionic field emission model. However, the resulting large number of parameters $\left(m^{*}, N_{D}\right.$, mean SB height value $\overline{\phi_{B n}}$ and standard deviation $\sigma_{\mathrm{s}}$ ) requires the independent determination of some of them.

For this purpose, room temperature $C-V$ measurements were performed to determine $N_{\mathrm{D}}$ and $\overline{\phi_{B n}}$. The $C-V$ dependence for SBs is described by the following equation [15]:

$C=\sqrt{\frac{q \epsilon_{\mathrm{S}} N_{\mathrm{D}}}{2\left(\phi_{\mathrm{B}, 0}-V-\xi-V_{\mathrm{t}}\right)}}$

An inverse square capacitance versus voltage characteristic is shown as filled squares in Fig. 4. For low reverse bias (from $-1 \mathrm{~V}$ to $0 \mathrm{~V}$ ) a straight line is observed. From the slope of this line the Si doping concentration can be estimated. A value of 1.32 $10^{18} \mathrm{~cm}^{-3}$ is obtained, corresponding to a resistivity of $0.019 \Omega$ $\mathrm{cm}$ which matches the specification of the Si substrate used $(0.01-0.02 \Omega \mathrm{cm})$. From its intercept on the voltage axis, the SB height is calculated to be $1.19 \mathrm{~V}$. This unusually high value can be attributed to the high current observed for the $\mathrm{Ni}-\mathrm{n}+\mathrm{Si}$ contacts that distorts the $C-V$ curve. As TE predicts a straight line in this diagram, this non-linear behavior confirms that this high current cannot be explained by considering only TE.

To overcome this issue, the results of the thermionic emission analysis of $\mathrm{Ni}-\mathrm{nSi}$ SBs can be used [9]. From $C-V$ measurements of a $\mathrm{Ni}-\mathrm{nSi} \mathrm{SB}$ (shown as circles in Fig. 4), a SB height of $0.76 \mathrm{~V}$ is obtained. This value should match the mean SB height $\overline{\phi_{B n}}$ of the model of Werner and Guttler. Also, from low-temperature $I-V$ measurements, a standard deviation of $66 \mathrm{mV}$ has been found. Using the $C-V$ measured value for $N_{\mathrm{D}}$ and the nSi results for $\overline{\phi_{B n}}$ and $\sigma_{\mathrm{s}}$, the $\mathrm{n}+\mathrm{Si}$ experimental data of Fig. 3 are fit by Eqs. (8) and (5), using $m^{*}$ as the only fitting parameter. A fit shown as a black line is obtained with $m^{*}=0.05$. This fit shows that the moderate decrease of $\left(J_{\text {TFE,f }}\right)$ with decreasing temperature observed in Figs. 2 and 3 is in agreement with the predictions for TFE through a spatially distributed SB height. For bulk Si, the theoretical value for $m^{*}$ in the direction of the tunneling current (transverse) is 0.19 . The low fitting value for the effective mass is an indication of crystal disorder at the $\mathrm{Ni}-\mathrm{Si}$ interface as such a disorder can drastically reduce the tunneling effective mass of electrons [16]. This fit is further discussed in Section 4.3, in comparison with reverse bias data.

While the domination of tunneling in reverse biased Schottky barriers has been widely acknowledged, the identification of TFE in forward bias is a novel result with particular importance. It has been pointed out that SBs need to be reverse biased for spin injection and detection, assuming domination of thermionic emission at forward bias [6]. Under this assumption a spin injection and detection device with two SBs in series and a single type semiconductor would not be possible. From our data, it emerges that the excess low forward bias current can be attributed to thermionic field emission through the SB. This corroborates experimental results showing spin dependent transmission at lowly forward biased FM/GaAs SBs [17].

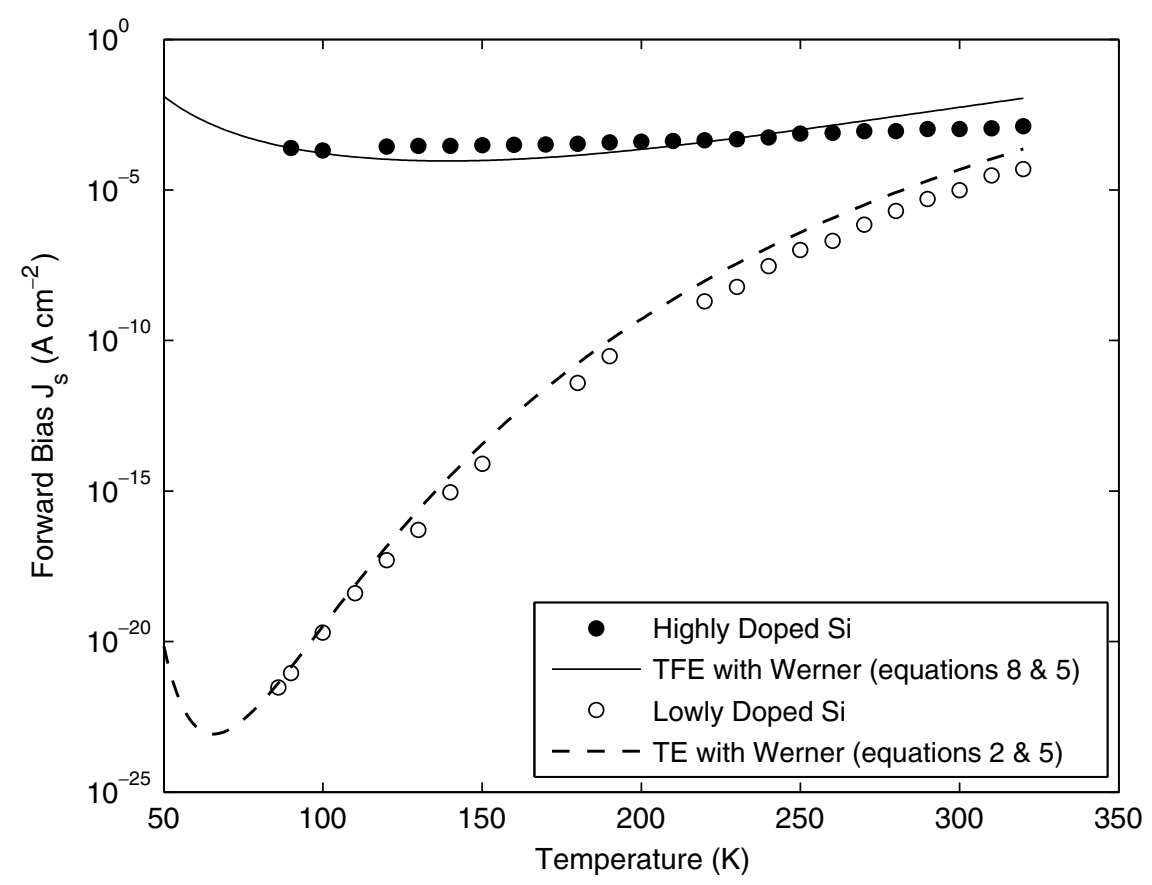

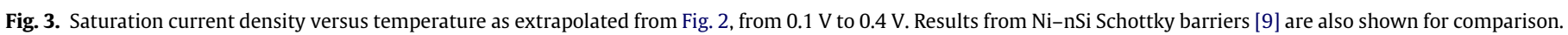




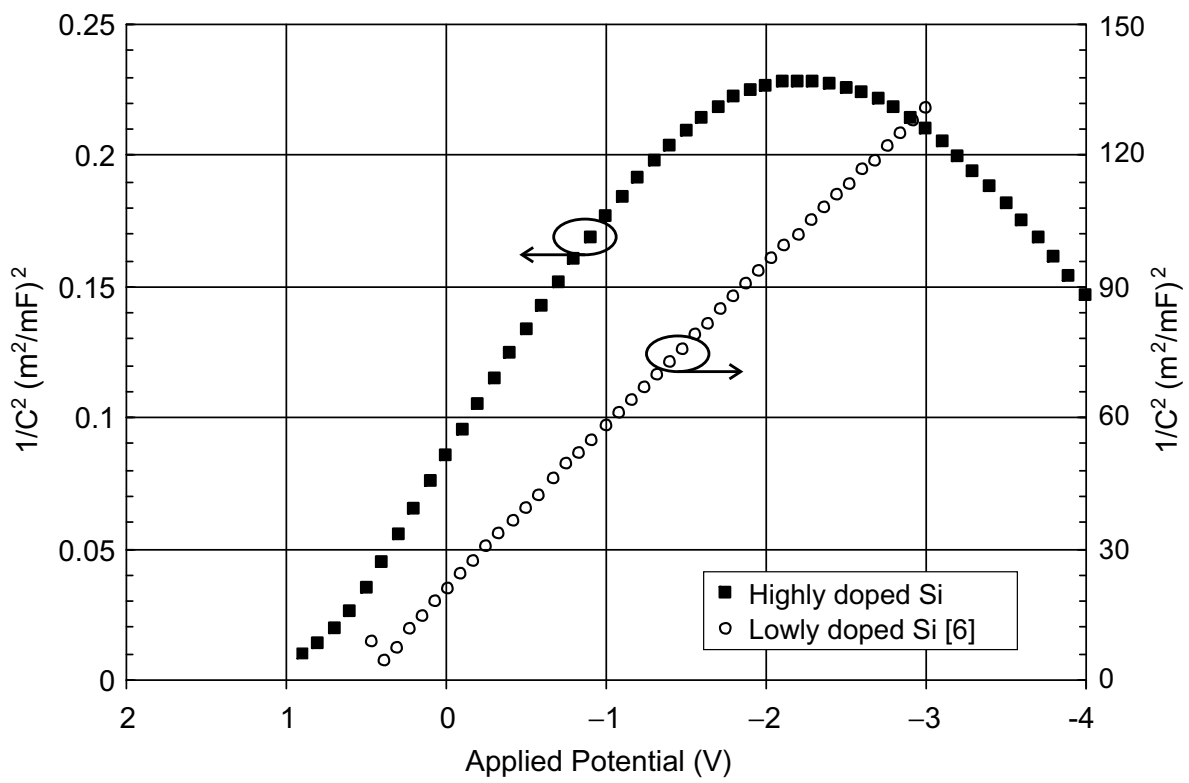

Fig. 4. $C-V$ measurements of electrodeposited $\mathrm{Ni}-\mathrm{Si}$ contacts for $\mathrm{n}+\mathrm{Si}$ and $\mathrm{nSi}$.

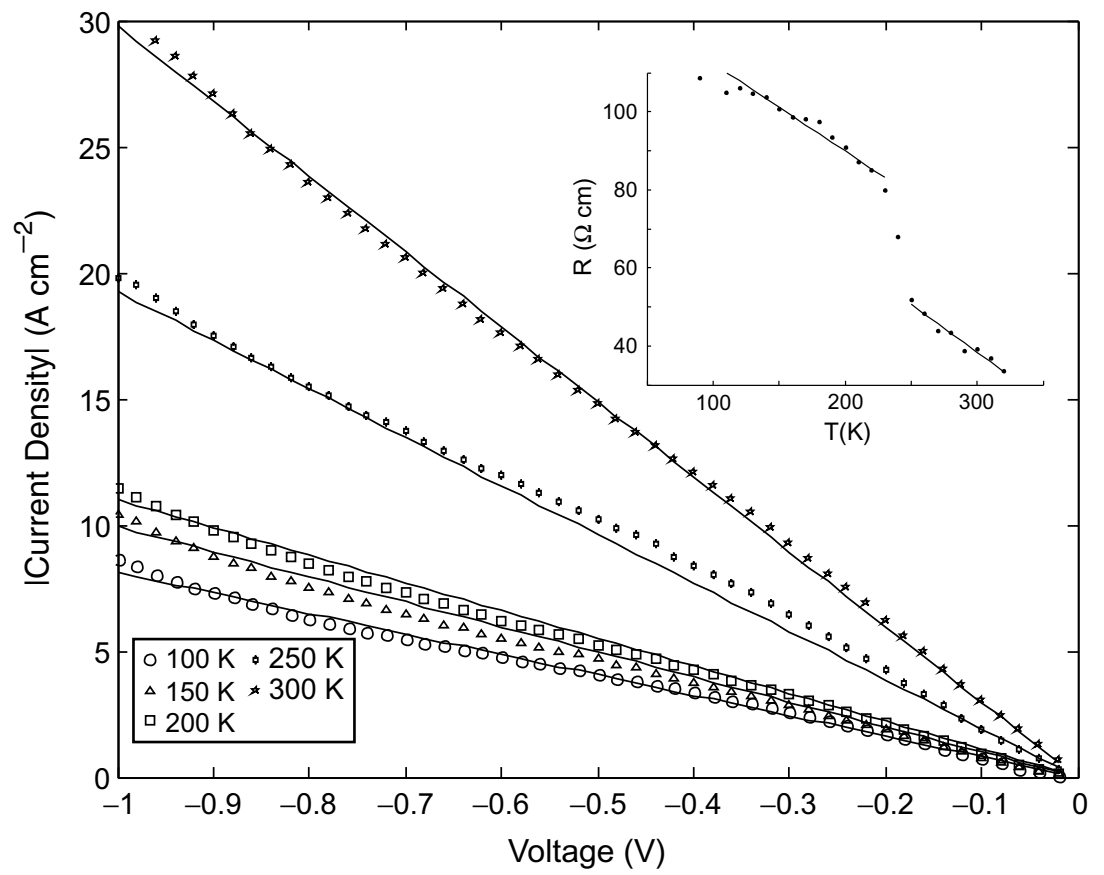

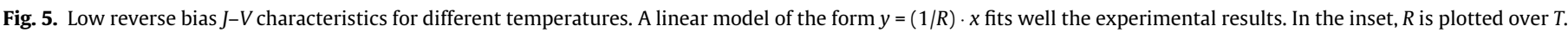

\subsection{Low reverse bias}

For low reverse bias, the current density appears to increase linearly with applied potential. Using a linear model, a good fit is obtained as illustrated in Fig. 5. The intersection of the fitting curves with the $J$ axis was set to zero. From this fit, the gradient can be extrapolated. The resulting values are plotted in the inset of Fig. 5. For low temperatures, the experimental results indicate a transport mechanism with small temperature dependence. For temperatures around $240 \mathrm{~K}$ a sharp peak of the temperature dependence is observed. This increase could be attributed to the activation of the high reverse bias mechanism (thermionic field emission) in this region for temperatures higher than $200 \mathrm{~K}$. A physical explanation of this linear behavior could involve tunneling effects through interface trap states at the Ni-Si contact.

\subsection{High reverse bias}

The response for reverse bias higher than $-1.5 \mathrm{~V}$ is studied by extrapolation of its saturation current density from the interval 


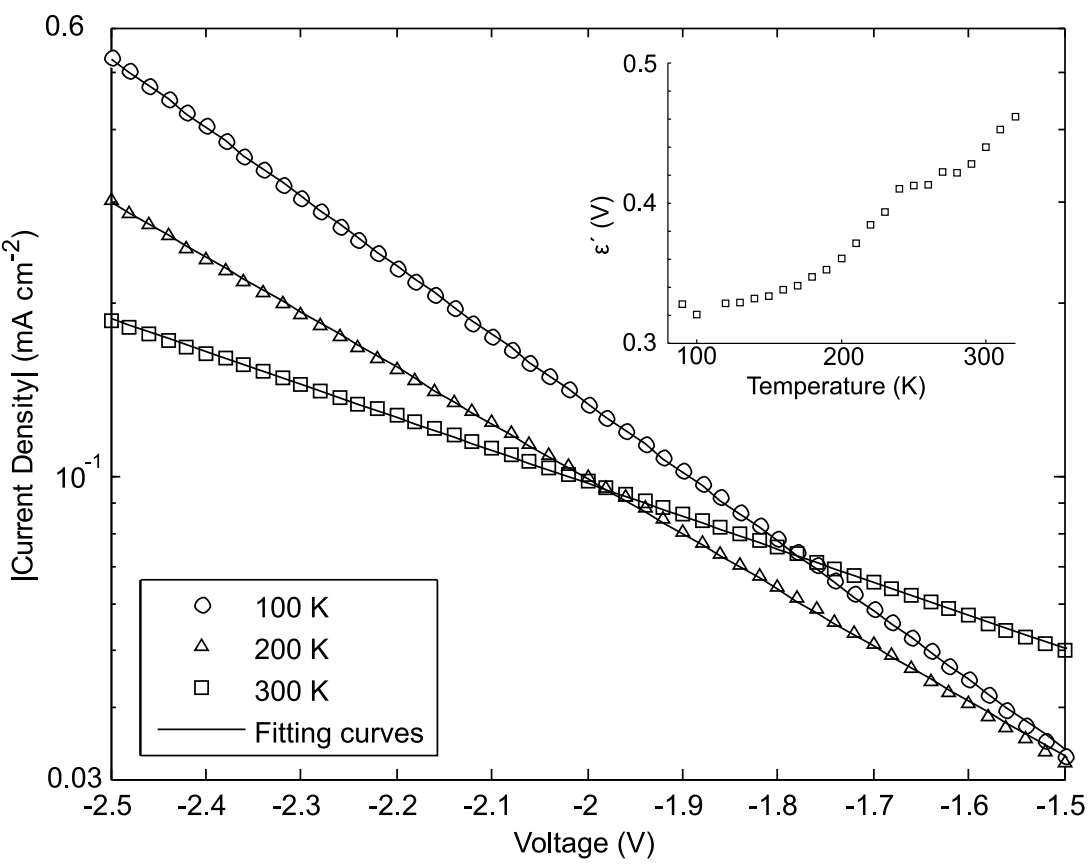

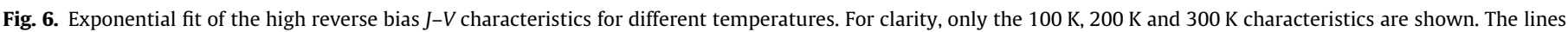
are linear extrapolations of the experimental data in the logarithmic scale. The extrapolated values of the inverse slope versus temperature are plotted in the inset.

between $-1.5 \mathrm{~V}$ and $-2.5 \mathrm{~V}$ as shown in Fig. 6. The excellent fit indicates that the high reverse bias current density increases exponentially with reverse voltage, in agreement with the predictions of thermionic field emission theory (Eq. (7)). The reverse bias slope $\varepsilon^{\prime}=\mathrm{d}(\ln J) / \mathrm{d} V$ and saturation current density $J_{\mathrm{s}, \mathrm{TFE}, \mathrm{r}}$ can be extracted from this fit.

The inverse slope $\left(\varepsilon^{\prime}\right)$ versus temperature is plotted in the inset of Fig. 6. $\varepsilon^{\prime}$ increases with increasing temperature. This is consistent with Eq. (11). The temperature dependence of $\varepsilon^{\prime}$ leads to the crossover observed in Fig. 2.
The extrapolated saturation current density divided by $T^{2}$ is plotted against $T$ in Fig. 7 (filled squares). For consistency, these results should be discussed in combination with the forward bias analysis. Therefore, the forward bias extrapolated data are plotted here for comparison (shown as filled circles in Fig. 7). It can be observed that the forward and reverse bias $J_{\mathrm{s}}$ follow similar trends.

The forward bias thermionic field emission fit as described in the analysis of the forward bias is shown as a solid line in Fig. 7. This corresponds to Eq. (8) with a temperature dependent barrier height as described in Eq. (5). Using the same values for all param-

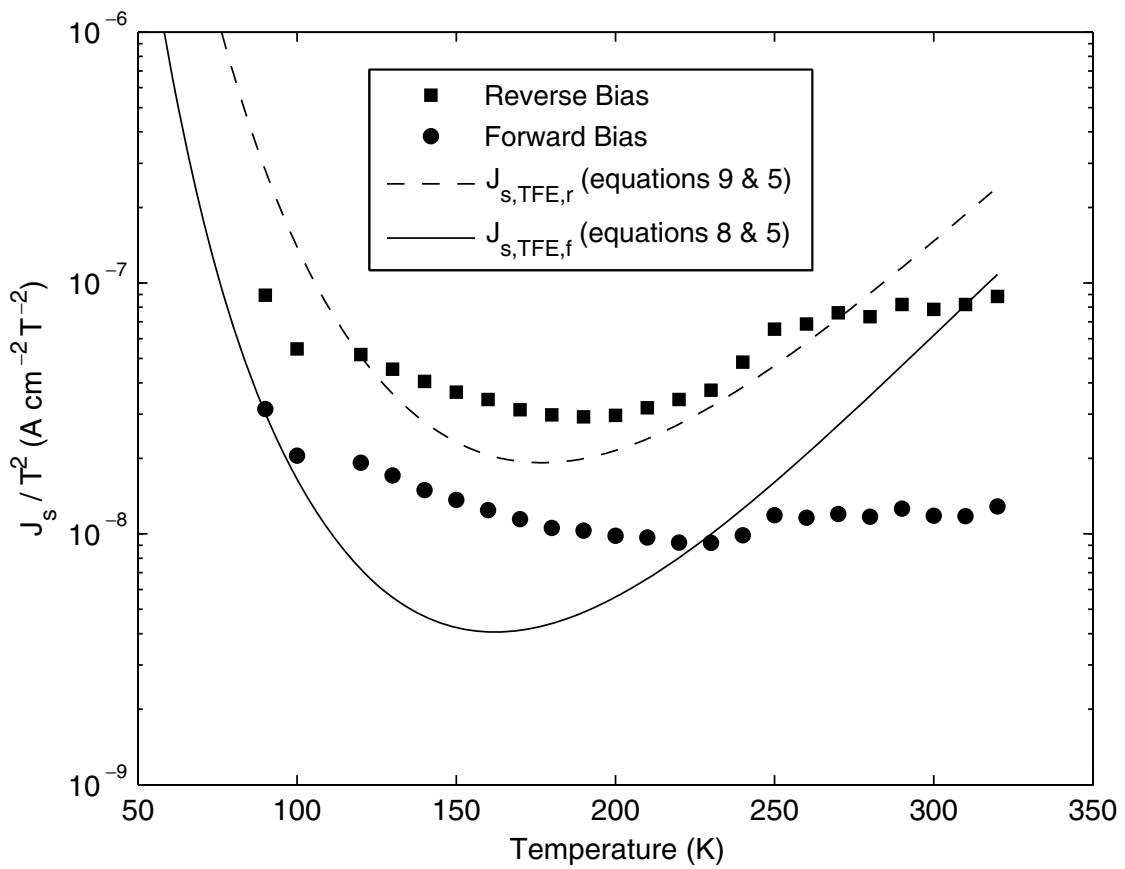

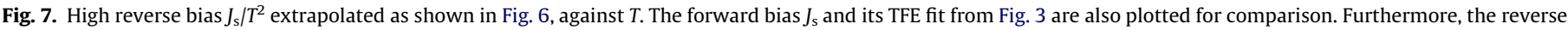
bias TFE model is plotted without any fitting parameters. 
eters, the reverse bias thermionic field emission saturation current density is calculated from Eqs. (9) and (5). The result is shown as a dashed line in Fig. 7. The pair of theoretical curves in Fig. 7 predict that the forward and reverse bias saturation current densities follow the same temperature-dependence trend. This is consistent with the experimental results which indeed exhibit a similarity analogous to the theoretical one. The qualitative matching of the reverse bias measurements to the TFE predictions is a considerable result as it is free of any fitting parameters. This is strengthened by the matching of the ratio of separate, extrapolated forward and reverse bias results to the theoretical predictions which is also achieved without any fitting parameters.

In the lower of temperatures appearing in Fig. 7 the validity of TFE is expected to weaken as field emission is starting to appear. Similarly, for the higher of temperatures in Fig. 7 thermionic emission is starting to play a role. This is the origin of the deviations from TFE observed at the lower and the upper temperature limits of the measurements.

\section{Conclusions}

In this work, current transport at electrodeposited $\mathrm{Ni}-\mathrm{n}+\mathrm{Si}$ contacts was studied. The cross-over of reverse bias TFE current-voltage characteristics for different temperatures predicted by Padovani and Stratton was experimentally demonstrated. The cross-over results in a reverse bias region with negative temperature coefficient of the TFE current. This effect is a consequence of the TFE theory for SBs which predicts a logarithmic current-voltage dependence in the reverse bias with slope that increases with decreasing temperature [15]. In addition, the results presented in this paper show tunneling domination at low forward bias. Such SBs could be used in series for all electrical spin injection and detection and research work toward this direction is underway.

\section{Acknowledgements}

This work was supported by EPSRC, the European Union (SINANO) and Innos Ltd who provided access to clean room processing.

\section{References}

[1] Schmidt G. Concepts for spin injection into semiconductors - a review. J Phys D Appl Phys 2005;38:R107-22.

[2] Jaffres H, Fert A. Spin injection from a ferromagnetic metal into a semiconductor. J Appl Phys 2002;91:8111-3.

[3] Hanbicki AT, van t Erve OMJ, Magno R, Kioseoglou G, Li CH, Jonker BT, et al. Analysis of the transport process providing spin injection through an $\mathrm{Fe} /$ AlGaAs Schottky barrier. Appl Phys Lett 2003;82:4092-4.

[4] van t Erve OMJ, Hanbicki AT, Holub M, Li CH, Awo-Affouda C, Thompson PE, et al. Electrical injection and detection of spin-polarized carriers in silicon in a lateral transport geometry. Appl Phys Lett 2007;91:212109.

[5] Bratkovsky AM, Osipov VV. Efficient spin injection and extraction in modified reverse and forward biased ferromagnetic-semiconductor junctions and lowpower ultrafast spin injection devices. Appl Phys A Mater Sci Proc 2005;80: 1237-46.

[6] Schmidt G, Molenkamp LW. Spin injection into semiconductors physics and experiments. Semicond Sci Technol 2002;17:310-21.

[7] Shannon JM. Thermionic-field emission through silicon Schottky barriers at room temperature. Solid-State Electron 1977;20:869-72.

[8] Lou X, Adelmann C, Crooker SA, Garlid ES, Zhang J, Reddy KSM, et al. Electrical detection of spin transport in lateral ferromagnet-semiconductor devices. Nat Phys 2007;3:197-202.

[9] Kiziroglou ME, Zhukov AA, Li X, Gonzalez DC, de Groot PAJ, Bartlett PN, et al. Analysis of thermionic emission from electrodeposited Ni-Si Schottky barriers. Solid-State Commun 2006;140:508-13.

[10] Padovani FA, Stratton R. Field and thermionic-field emission in Schottky barriers. Solid-State Electron 1966;9:69.

[11] Rhoderick EH, Williams RH. Metal-semiconductor contacts. second ed. Oxford: Clarendon Press; 1988

[12] Werner JH, Guttler HH. Barrier inhomogeneities at Schottky contacts. J Appl Phys 1991;69(3):1522-33.

[13] Bartlett PN, Ghanem MA, Hallag ISE, de Groot P, Zhukov A. Electrochemical deposition of macroporous magnetic networks using colloidal templates. J Mater Chem 2003(13):2596-602.

[14] Kiziroglou ME, Zhukov AA, Abdelsalam M, Li X, de Groot PAJ, Bartlett PN, et al. Electrodeposition of Ni-Si Schottky barriers. IEEE Trans Magn 2005;41(10): 2639-41.

[15] Sze SM. Physics of semiconductor devices. Wiley Interscience; 1981.

[16] Shannon JM, Nieuwesteeg KJBM. Tunneling effective mass in hydrogenated amorphous silicon. Appl Phys Lett 1993;62:1815-7.

[17] Steinmuller SJ, Gurtler CM, Wastlbauer G, Bland JAC. Separation of electron spin filtering and magnetic circular dichroism effects in photoexcitation studies of hybrid ferromagnet/GaAs Schottky barrier structures. Phys Rev B 2005;72:045301. 